\title{
Distinct Immunosuppressive Effect by Isodon serra Extracts
}

By: Yan Zhang, Jianwen Liu, Wei Jia, AiHua Zhao, and Ting Li

Zhang, Y., Liu, J., Jia, W. Zhao, A., \& Li, T. (2005). Distinct immunosuppressive effect by Isodon serra extracts. International Immunopharmacology, 5, 1957-1965.

\author{
***Note: This version of the document is not the copy of record. Made available courtesy of \\ Elsevier. Link to Journal:/http://www.journals.elsevier.com/international- \\ immunopharmacology/ \\ Link to Article:[http://www.sciencedirect.com/science/article/pii/S1567576905001803
}

\begin{abstract}
:
Distinct effect of ent-Kaurene Diterpenoids from Isodon serra on abnormal proliferation of murine lymphocytes was examined with MTT assay and Flow Cytometry Analyses (FCAS). After choosing the most appropriate monomer from these Diterpenoids, we introduced mouse tumescence model to investigate whether it could impact cytokine production in vivo with ELISA assay.
\end{abstract}

The result of MTT assay showed that four ent-Kaurene Diterpenoids could effectively suppress the murine splenic T lymphocytes overproduction stimulated by Concanavalin A, while inhibitive effect was softer on normal sleep lymphocytes than the stimulated ones. Among four ent-Kaurene Diterpenoids, Enmein was the most sensitive one with $\mathrm{IC}_{50} / \mathrm{EC}_{50}$ equaling to 1.55 . This inhibitive activity was due to interfering DNA replication in G1-S stage and to regulating cell cycle according to flow cytometry analyses (FCAS) result. Xylene-induced mouse tumescence model result further suggested that Enmein depressed the murine ear swelling extent and the level of Interleukin-2 in blood serum in a dose-dependent manner.

In conclusion, it demonstrated that four ent-Kaurene Diterpenoids from I. serra had distinct immunosuppressive effect in vitro and in vivo systems, which primarily differentiated Enmein from the others. The experimental results provided insight into a potential immunosuppressive action of Enmein as a promising drug, though profound mechanism remained to be further studied.

Article:

INTRODUCTION

For over hundreds of years, traditional Chinese doctors have accumulated and developed considerable pharmacopoeias that are largely used in modern China and many other parts of the world [1]. Isodon serra (MAXIM.) HARA, named Xi Huangcao in Chinese, is distributed in Southeast area of P.R. China. As a perennial herb, it has been popularly used for treatment of arthritis, enteritis, jaundice, hepatitis, lepromatous leprosy, ascariasis and acute cholecystitis [2]. 
It is known that cyclophosphamide is an antitumor medicine which also has immunosuppressive activity. Correspondingly, I. serra monomers were reported to vary in efficacy especially for anti-tumor biological activity [3] and [4]. In our study, we aimed to explore other functions like immunosuppressive activity of these $I$. serra extracts besides their antitumor activity [5].

Recently, our studies [6] on I. serra extracts have led to the isolation of some novel ent-Kaurene Diterpenoids, which showed molecular components and potential inhibitive activities in $\mathrm{T}$ lymphocytes proliferation. However, detailed comparison of inhibitive activities among these extracts has not been reported before. So major objective of our study is to differentiate the most immunosuppressive compound from these ent-Kaurene Diterpenoids both in vitro and in vivo experimental models.

\section{MATERIALS AND METHODS}

\section{Reagents}

Four ent-Kaurene Diterpenoids of I. serra were extracted and purified by school of Pharmacy, Shanghai Jiao Tong University, P.R. China [6]. Chemical structures of these ent-Kaurene Diterpenoids were shown in Fig. 1. Purity of these ent-Kaurene Diterpenoids was over 98\%. These extracted monomers were respectively observed for their pharmaceutical efficiency in the following experimental models.

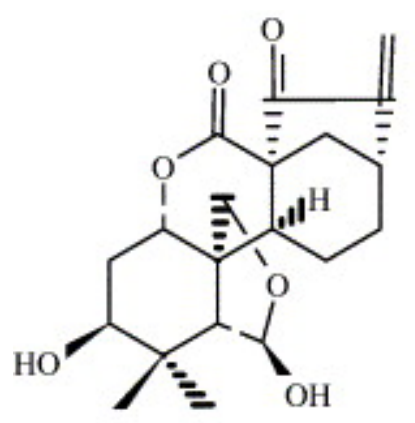

Enmein

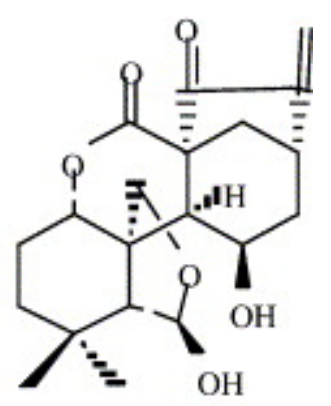

Nodosin

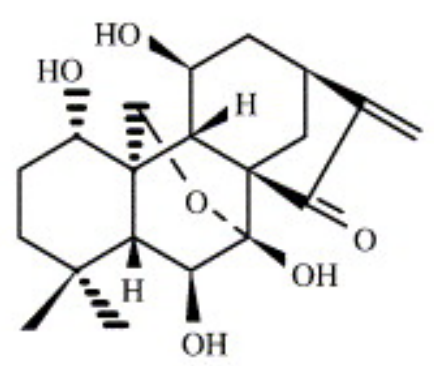

Lasiodonin

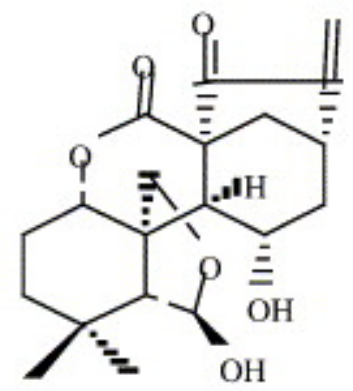

Epinodosin

Figure 1: Chemical structures of ent-Kaurene Diterpenoids from Isodon serra.

It was reported that multi-glycoside from Tripterygium wilfordii Hook f. (GTW) was used for various immune and inflammatory diseases in China [7] and [8]. GTW had suppressive effects on acute inflammatory changes most likely by reducing expression of injurious cytokines [9]. Here 
GTW (obtained from Fujian-Huitian Biological Pharmacy Co.) was used as the positive reference substance in vitro models. Since tacrolimus (FK506) was a clinical drug with great immunosuppressive activity [10], FK506 (obtained from Fujisawa Ireland Ltd.) was designed as positive group in mouse tumescence model.

\section{Animals}

Pathogen-free female/male BALB/c mice weighing $20 \pm 2$ g, 8-10 weeks of age, were purchased from ShangHai Center of Experimental Animals, Chinese Academy of Sciences. License number: SCXK (Shanghai) 2003-0003.The animals were fed in our SPF standards animal laboratory with $24 \pm 2{ }^{\circ} \mathrm{C}$ and $50 \%$ humidity, and with a 12-h light/dark cycle. They were provided with sterilized food and water. They would not be applied to our experiment until they were fed for 1 week obtained from ShangHai Center of Experimental Animals.

\section{Splenocyte isolation}

Fresh lymphocyte suspensions were prepared before each experiment from male BALB/c mice. They were exsanguinated, and then spleens were immediately excised. Cells derived from spleens sequentially passed through a 100-mesh screen to harvest for mononuclear lymphocytes suspension. Erythrocytes were lysed with Gey's reagent for 5 min [11]. Cells were then washed with sterile phosphate buffered saline (PBS). Mononuclear lymphocytes were isolated by buoyant density centrifugation (5 min at $1000 \mathrm{rpm}$ ). After that, the isolated splenic lymphocytes were resuspended at $1 \times 10^{6}$ cells/ml in RPMI-1640 medium (Sigma Co.) supplemented with $10 \%$ (v/v) heat-inactivated bovine serum (GIBCO Co.). Cells were cultured in 96 well tissue culture plates with $2 \times 10^{5}$ lymphocytes per well [12]. They were incubated at $37^{\circ} \mathrm{C}$ in a humidified atmosphere of $5 \%$ carbon dioxide $\left(\mathrm{CO}_{2}\right)$ for the indicated period. The recovered cells were typically $>98 \%$ viable as assessed by Trypan blue exclusion, and the heterogeneous mononuclear cell suspension mainly consisted of $40 \%$ B cells and $60 \%$ T cells.

\section{Cell viability}

3-(4,5-Dimethylthiazol-2-yl)-2,5-diphenyl tetrazolium bromide (MTT) (Gibico) was diluted to 5 $\mathrm{mg} / \mathrm{ml}$ in PBS and filtered once through a $0.22-\mu \mathrm{m}$ filter. The diluted MTT was under protected from light at $4{ }^{\circ} \mathrm{C}$ and used within a month. MTT colorimetric assay was applied to study cytotoxicity of ent-Kaurene Diterpenoids from I. serra.

Briefly, isolated splenocytes $(100 \mu \mathrm{l} /$ well) were cultured in 96 well tissue culture plates in the presence of these compounds separately. MTT was added after $48 \mathrm{~h}$ at a final concentration of $500 \mu \mathrm{g} / \mathrm{ml}$ and incubated for $4 \mathrm{~h}$. After removal of MTT, the formazan precipitate was solubilized in DMSO (100 $\mu \mathrm{l} /$ well) and measured on a microplate reader model 550(Bio-rad, California, USA) at the absorbance wavelength of $570 \mathrm{~nm}$ and reference wavelength of $655 \mathrm{~nm}$. The experiment was repeated three times. Viability rate of cells = average OD of test team / average OD of control team $\times 100 \%$. Half inhibitive concentration $\left(\mathrm{IC}_{50}\right)$ here meant the drug concentration when viability rate of cells reached $50 \%$.

\section{Excessive multiplication response}

Murine splenic lymphocytes were aliquotted in 96-well plates $(100 \mu \mathrm{l} /$ well $)$ and stimulated by Concanavalin A (ConA) (Sigma). Meanwhile ent-Kaurene Diterpenoids were added. GTW took the positive groups. The lymphocytes survival was measured with MTT assay. The experiment 
was repeated three times. Viability rate of T-lymphocytes = average OD of test team / average OD of control team $\times 100 \%$. Half effective concentration $\left(E_{50}\right)$, which was employed as the activity evaluating parameter, here meant the drug concentration when the viability rate of Tlymphocytes equaled $50 \%$.

\section{Flow cytometry analysis}

Flow cytometry analysis was applied to investigate whether these monomers had DNA synthesis interdiction of activated T-lymphocytes [13]. Briefly, ConA-induced cells were cultured with Enmein or GTW. After $48 \mathrm{~h}$, cells were trypsinized to form single-cell suspension, and were diluted to $1 \times 10^{6}$ cells $/ \mathrm{ml}$. After rinsed twice with cold PBS, cells were fixation with PBS containing $70 \%$ of methanol for $1 \mathrm{~h}$ at $4{ }^{\circ} \mathrm{C}$. Subsequently they were treated with Rnase A for 30 min and then with Trypsin $(0.5 \% \mathrm{w} / \mathrm{v})$ and EDTA $(0.2 \% \mathrm{w} / \mathrm{v})$ for $5 \mathrm{~min}$. Finally, cells were stained with propidium iodide (PI) and analysed in a FACScan flow cytometer. We analyzed 10,000 events for each sample.

\section{Mouse tumescence model}

Ear-swelling response in murine was an experimental animal model, which was concerned with the recruitment and the activation of immune and inflammatory cells [14] and [15]. This tumescence model had advantages of little difference, high sensitivity and repeatability, etc. BALB/c mice, 8-10 weeks of age, were used for this experimental animal model. Briefly, male/female BALB/c mice were separately divided into several groups. Enmein was separately injected into mice at the dose of $6.52 \mathrm{mg} / \mathrm{kg}$ (LOW), $9.78 \mathrm{mg} / \mathrm{kg}$ (MID) or $13.04 \mathrm{mg} / \mathrm{kg}$ (HIGH) for 5 days; in parallel, mice treated with tacrolimus (FK506) $(1 \mathrm{mg} / \mathrm{kg}$ ) were divided as positive group. These doses were all conversion quantities of clinical dosages. On the 5 th day, $1 \mathrm{~h}$ after administering, we injected xylene into the left ear of each mouse. Forty minutes later, mice blood specimens of Enmein-treated groups were collected and stored at $-20^{\circ} \mathrm{C}$. After the mice were executed, murine ear weight was then quantified.

To evaluate pharmaceutical efficacy, ear tumescence extent was calculated as follows: Degree of ear swelling $=\left(M_{\text {left ear }}-M_{\text {right ear }}\right) / M_{\text {mouse }} . M_{\text {left ear }}$ indicated net weight of each left murine ear; $M_{\text {right ear }}$ indicated net weight of each right murine ear; $M_{\text {mouse }}$ indicated weight of each mouse.

\section{Enzyme-linked immunosorbent assay (ELISA)}

The production of cytokine (Interleukin-2) in blood serum (collected before) was measured by a standard sandwich cytokine ELISA procedure to assess the pharmaceutical effect on cytokine quantity in vivo model. Standards (recombinant cytokine at 0, 15.63, 31.25, 62.5, 125, 250, 500, $1000 \mathrm{pg} / \mathrm{ml}$ concentrations) and samples were added in $100 \mu \mathrm{l} /$ well. Cytokine quantities in the samples were calculated from standard curves of recombinant cytokines using a regression linear method. The assay was performed with manufacturer's instructions of mouse Interleukin-2 kits from R\&D systems (Minneapolis, MN). Absorbance results were assessed using an ELISA microplate reader set (Multiskan Ex, Lab systems, Finland).

\section{Statistical analysis}

Statistical analysis was performed using the Origin 7.0 software to evaluate the significance of differences between groups. In all these graphs, * indicated significantly different than untreated groups, $p<0.05$; ** indicated significantly different than untreated groups, $p<0.01$; *** 
indicated significantly different than untreated groups, $p<0.001$. All data points represented the mean of triplicates.

\section{RESULTS}

\section{Cytotoxicity evaluation of I. serra extracts in vitro}

Splenic lymphocytes $\left(1 \times 10^{6}\right.$ cells $\left./ \mathrm{ml}\right)$ were treated with $I$. serra extracts at different concentrations $(3.13,6.25,12.5,25 \mu \mathrm{g} / \mathrm{ml})$ in $48 \mathrm{~h}$. These extracts were measured by MTT method that exhibited different cytotoxicity. In Fig. 2a, drug concentration was taken for horizontal coordinates, and cells viability rate for vertical coordinates. The result showed that the inhibitive activity of Enmein on lymphocytes with highest viability rate was much lower than of Lasiodonin, Nodosin and Epinodosin. Within concentration from $6.25 \mu \mathrm{g} / \mathrm{ml}$ to $25 \mu \mathrm{g} / \mathrm{ml}$, the inhibitive activity of Enmein on lymphocytes appeared statistical significant difference $(p<0.001)$. Moreover, GTW (positive reference substance) showed slight inhibitive effect on lymphocytes. Most data here showed statistical differences $(p<0.01)$.
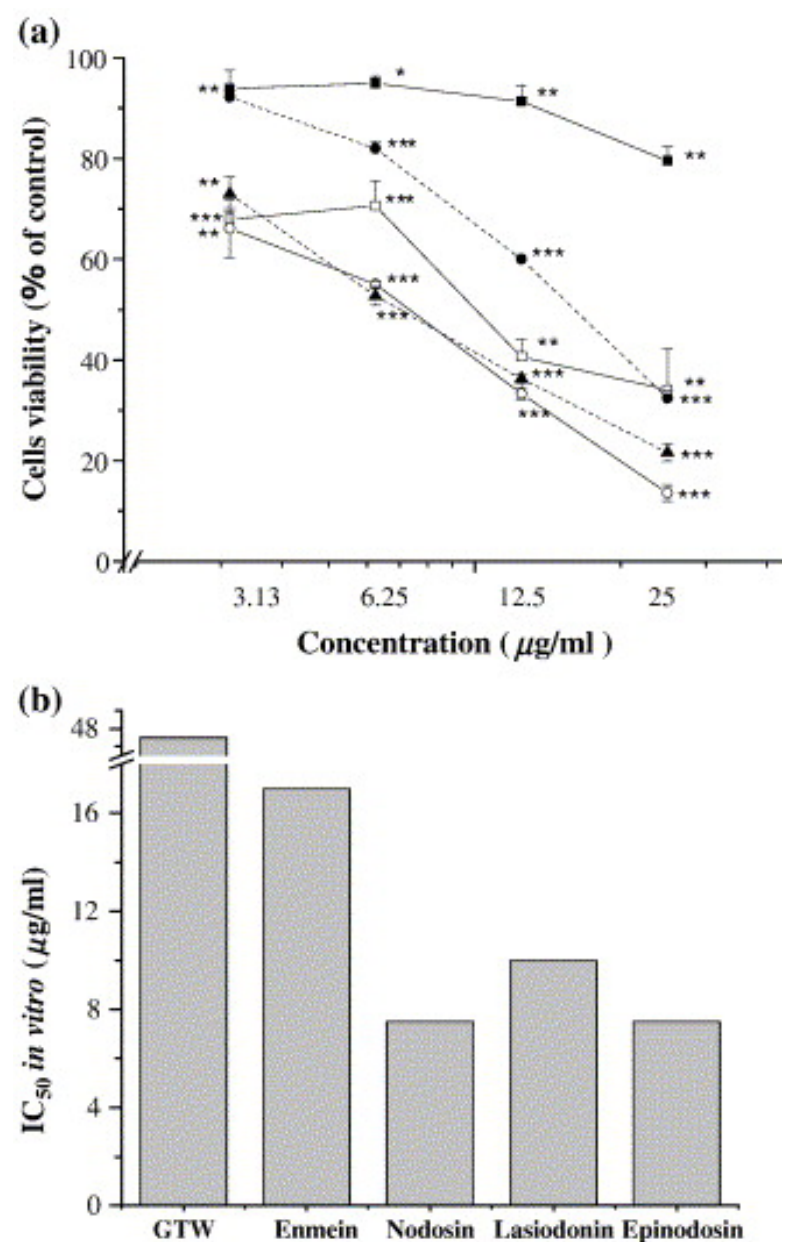

Figure 2: Cytotoxicity evaluation of Isodon serra extracts in vitro. (a) Cells were cultured with various

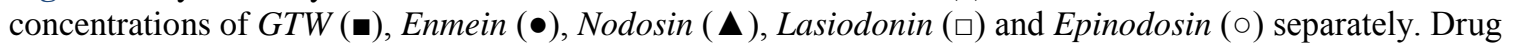
concentration was taken for horizontal coordinates, and cells viability rate for vertical coordinates. The inhibition of Enmein with highest viability rate was much lower than that of Lasiodonin, Nodosin and Epinodosin. From 6.25 $\mu \mathrm{g} / \mathrm{ml}$ to $25 \mu \mathrm{g} / \mathrm{ml}$, the activity of Enmein on lymphocytes showed statistical significant difference $(p<0.001)$. (b) Each column represented Half-inhibiting concentration $\left(\mathrm{IC}_{50}\right)$ of corresponding drug on normal lymphocytes. IC 50 of Enmein was the highest among four extracts, reaching $17 \mu \mathrm{g} / \mathrm{ml}$. And $\mathrm{IC}_{50}$ of Lasiodonin came to $10 \mu \mathrm{g} / \mathrm{ml}$. $\mathrm{IC}_{50}$ 
for each I. serra extract showed the following order: Enmein $>$ Lasiodonin $>$ Nodosin $>$ Epinodosin. ${ }^{*}$ Indicated significant difference between control group and experimental group, $p<0.05$; $* *$ indicated significant difference between control-induced group and experimental group, $p<0.01$; ***indicated significantly difference controlinduced group and experimental group, $p<0.001$.

Half inhibitive activity $\left(\mathrm{IC}_{50}\right)$ for each $I$. serra extract was estimated based on results in Fig. 2a. In Fig. 2b, each column represented $\mathrm{IC}_{50}$ of corresponding compound. The $\mathrm{IC}_{50}$ of Enmein was highest among four extracts, reaching $17 \mu \mathrm{g} / \mathrm{ml}$. Meanwhile, the $\mathrm{IC}_{50}$ of Lasiodonin came to 10 $\mu \mathrm{g} / \mathrm{ml}$. The $\mathrm{IC}_{50}$ of these extracts showed the following order:

Enmein > Lasiodonin > Nodosin > Epinodosin (Fig. 2b).

\section{Inhibitive activity evaluation to over-activated $T$ lymphocytes}

ConA-induced lymphocytes $\left(1 \times 10^{6}\right.$ cells/ml) were treated with various concentrations of $I$. serra extracts in the range of 3.13-25 $\mu \mathrm{g} / \mathrm{ml}$ in $48 \mathrm{~h}$. The concentration of ConA was held constant at $3 \mu \mathrm{g} / \mathrm{ml}$. Then they were measured by MTT method. In Fig. 3a, from 3.13 to 25 $\mu \mathrm{g} / \mathrm{ml}$, the viability rate of Nodosin-treated lymphocytes went down $75 \%$ and to $5 \%$ at $48 \mathrm{~h}$, while the viability rate of Enmein-treated cells went to 20\%. Moreover, GTW (positive reference substance) also showed significant inhibitive activity on lymphocytes. Most data here appeared statistical significant difference $(p<0.001)$.

Half effective concentration $\left(\mathrm{EC}_{50}\right)$ of $I$. serra extracts was estimated based on results in Fig. 3a. In Fig. 3b, each column represented $\mathrm{EC}_{50}$ of corresponding compound to ConA-induced murine splenic T lymphocytes. The $\mathrm{EC}_{50}$ of Nodosin was the lowest in four extracts, reaching $5.5 \mu \mathrm{g} / \mathrm{ml}$. Meanwhile, the $\mathrm{EC}_{50}$ of Enmein reached $11 \mu \mathrm{g} / \mathrm{ml}$. The $\mathrm{EC}_{50}$ of these extracts showed the following order: Nodosin $<$ Epinodosin $<$ Enmein $<$ Lasiodonin.

Designated as a therapeutic index, the ratio of $\mathrm{IC}_{50}$ versus $\mathrm{EC}_{50}$ was estimated for each target compound based on results in Fig. 2 and Fig. 3. When counting up above-mentioned results, the ratio of these extracts showed the following order:

Enmein > Nodosin > Epinodosin > Lasiodonin successively (Fig. 3c). Among four ent-Kaurene Diterpenoids, Enmein was the most sensitive one with $\mathrm{IC}_{50} / \mathrm{EC}_{50}$ equaling to 1.55.

In conclusion, according to ConA-induced T lymphocytes proliferation model (Fig. 3c), Enmein was the most excellent ent-Kaurene Diterpenoids from I. serra. They had all shown inhibitive activity to cells over-proliferation and primarily immunosuppressive activity. Consequently, we carried out further research around Enmein by flow cytometry analysis. 
(a)
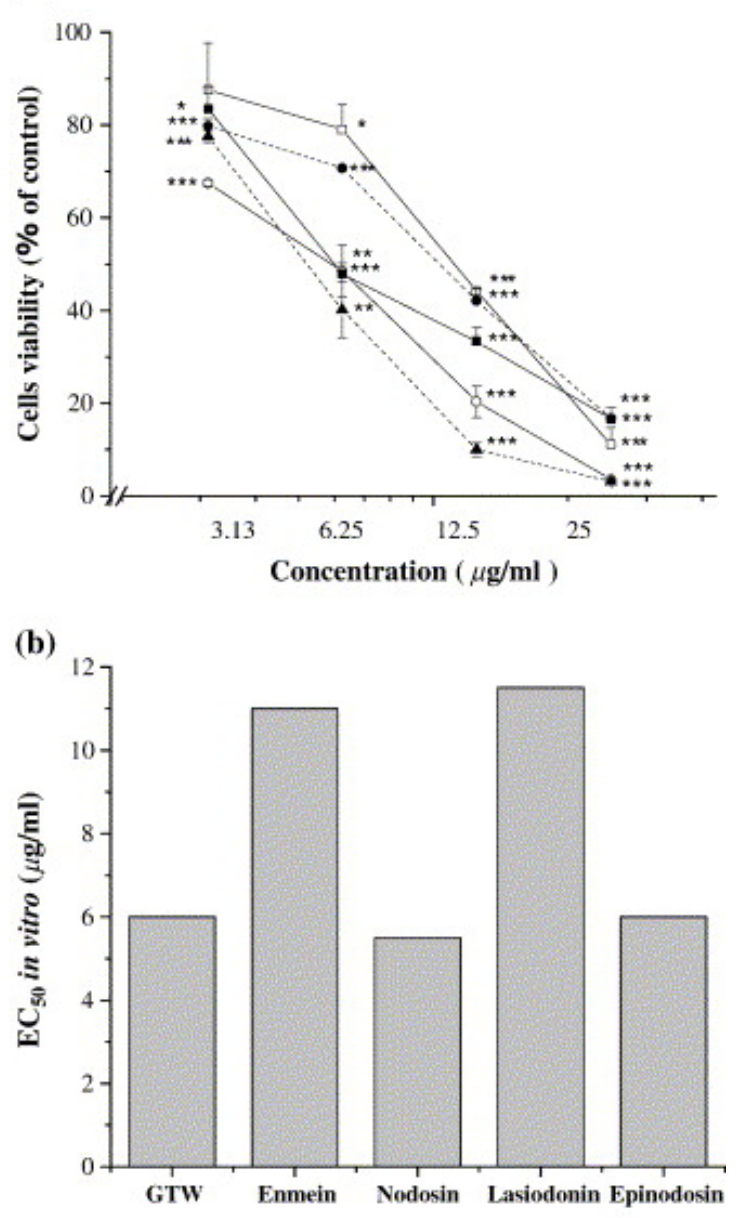

(c)

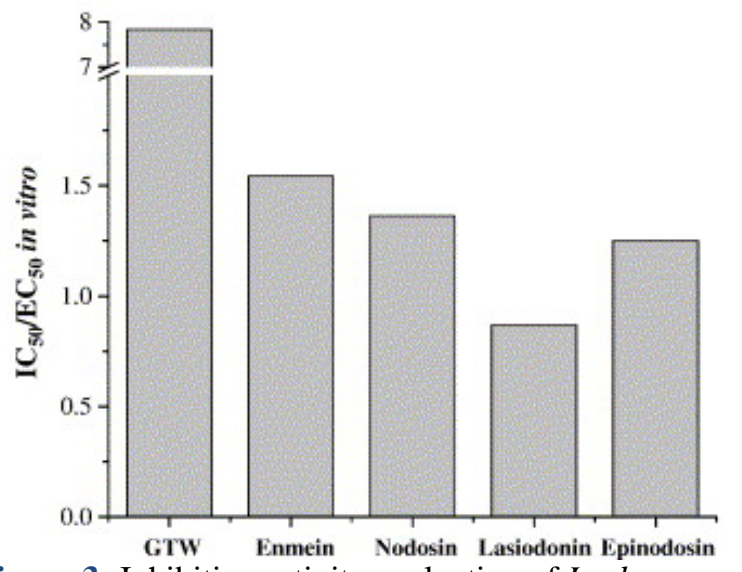

Figure 3: Inhibitive activity evaluation of Isodon serra extracts on over-activated T lymphocytes. (a) Splenic lymphocytes were cultured with ConA in the presence of various concentrations of GTW (•), Enmein $(\bullet)$, Nodosin (ム), Lasiodonin ( $\square$ ) and Epinodosin ( $\circ$ ) for $48 \mathrm{~h}$ and quantitated by MTT assay. Drug concentration was taken for horizontal coordinates, and cells viability rate for vertical coordinates. The concentration of each compound was varied as indicated (units: $\mu \mathrm{g} / \mathrm{ml}$ ). Between $3.13 \mu \mathrm{g} / \mathrm{ml}$ and $25 \mu \mathrm{g} / \mathrm{ml}$, the result indicated that the viability rate of Nodosin-treated lymphocytes was down from $75 \%$ and to $5 \%$ at 48 h, when the viability rate of Enmein-treated cells remained 20\%. Most data here appeared statistical significant difference $(p<0.001)$. (b) Each column represented half effected concentration $\left(\mathrm{EC}_{50}\right.$ ) from different $I$. serra extract by ConA-induced lymphocytes. The $\mathrm{EC}_{50}$ of Nodosin was the lowest in four extracts, reaching $5.5 \mu \mathrm{g} / \mathrm{ml}$. Meanwhile, the $\mathrm{EC}_{50}$ of Enmein reached $11 \mu \mathrm{g} / \mathrm{ml}$. The 
$\mathrm{EC}_{50}$ for I. serra extracts showed the following order: Nodosin $<$ Epinodosin $<$ Enmein $<$ Lasiodonin. (c) The ratio of $\mathrm{IC}_{50}$ versus $\mathrm{EC}_{50}$ designated as a therapeutic index was estimated for each target compound based on results in Figs. $2 \mathrm{~b}$ and part (b) of this figure. Counting up above-mentioned results, the ratio of $\mathrm{IC}_{50}$ versus $\mathrm{EC}_{50}$ for $I$. serra extracts showed the following order: Enmein > Nodosin > Epinodosin > Lasiodonin successively. Among four entKaurene Diterpenoids, Enmein was the most sensitive one with $\mathrm{IC}_{50} / \mathrm{EC}_{50}$ equaling to 1.55 . *Indicated significant difference between ConA-induced group and experimental group, $p<0.05$; **indicated significant difference between ConA-induced group and experimental group, $p<0.01$; ***indicated significantly difference ConAinduced group and experimental group, $p<0.001$.

Interference effect on cell cycle by flow cytometry analysis

Results above indicated that the optimal concentration of Enmein was higher than $\mathrm{EC}_{50}$ and lower than IC $_{50}(11-17 \mu \mathrm{g} / \mathrm{ml})$. According to this, we affirmed the middle dose at $11 \mu \mathrm{g} / \mathrm{ml}$ (MID), the low dose at $5.5 \mu \mathrm{g} / \mathrm{ml}$ (LOW), and the high dose at $22 \mu \mathrm{g} / \mathrm{ml}$ (HIGH). We performed activity of Enmein on cell cycle by Flow cytometry analysis (Fig. 4). ConA-induced T lymphocytes were treated with Enmein at $5.5 \mu \mathrm{g} / \mathrm{ml}$ (LOW), $11 \mu \mathrm{g} / \mathrm{ml}$ (MID) or $22 \mu \mathrm{g} / \mathrm{ml}$ (HIGH) or with $G T W$ at $11 \mu \mathrm{g} / \mathrm{ml}$ (MID) or $22 \mu \mathrm{g} / \mathrm{ml}$ (HIGH) in $48 \mathrm{~h}$, making normal lymphocytes as a control.

The results of FCAS assay (Fig. 4a) indicated that after lymphocytes were exposed to Enmein for $48 \mathrm{~h}$, percentage of $\mathrm{G}_{1}$ stage cells increased from $78 \%$ to $93 \%$ that was equal to normal control group (93\%). Results in Fig. 4b showed that Enmein could mainly cause the percentage of S stage cells decreased from $19 \%$ to $6 \%$ that was equal to normal control group (6\%). The data in Fig. 4c obviously indicated that Enmein could mainly cause the percentage of $\mathrm{G}_{2} / \mathrm{M}$ stage cells to decrease from $2 \%$ to $0.4 \%$ which was equal to normal control group $(0.58 \%)$.

\section{Effect on mouse xylene-induced ear tumefaction}

The degree of tumefaction was a useful marker for autoimmune activation and also played a crucial role in triggering activation of inflammatory [14] and [15]. Here we adopted xyleneinduced murine ear tumefaction model to investigate the immunosuppressive effect of Enmein in vivo. The result (Fig. 5a and b) indicated that the xylene-induced ear swelling was evidently inhibited both in female and male mice treated with FK506 $(1 \mathrm{mg} / \mathrm{kg})$ or Enmein at the dose of $6.52 \mathrm{mg} / \mathrm{kg}$ (LOW), $9.78 \mathrm{mg} / \mathrm{kg}$ (MID) or $13.04 \mathrm{mg} / \mathrm{kg}$ (HIGH) for 5 days. Compared with the control group, significantly inhibitive activity was noticed in Enmein groups, in a dosedependant manner especially for male mice. 

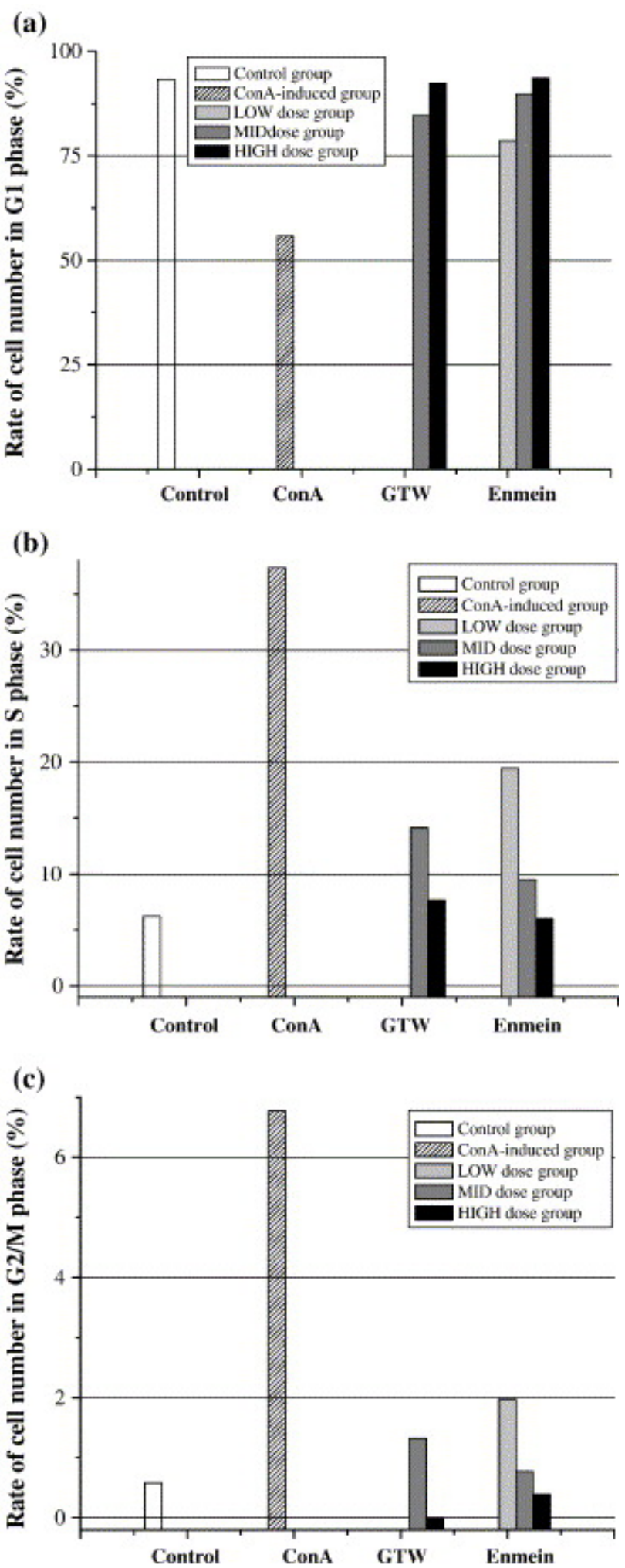

Figure 4: Interdiction effect of Enmein on cell cycle by flow cytometry analysis. ConA-induced lymphocytes were treated with Enmein at $5.5 \mu \mathrm{g} / \mathrm{ml}$ (LOW), $11 \mu \mathrm{g} / \mathrm{ml}$ (MID) or $22 \mu \mathrm{g} / \mathrm{ml}$ (HIGH) or GTW at $11 \mu \mathrm{g} / \mathrm{ml}$ (MID) or 22 $\mu \mathrm{g} / \mathrm{ml}$ (HIGH) in $48 \mathrm{~h}$, making normal lymphocytes as a control. (a) The results of FCAS assay indicated that after lymphocytes were exposed to Enmein for $48 \mathrm{~h}$, the percentage of $\mathrm{G}_{1}$ stage cells increased from $78 \%$ to $93 \%$ that was equal to normal control group (93\%). (b) The chart obviously showed that Enmein could mainly cause the 
percentage of S stage cells decreased from $19 \%$ to $6 \%$ that was equal to normal control group (6\%). (c) The data indicated that Enmein could mainly cause the percentage of $\mathrm{G}_{2} / \mathrm{M}$ stage cells decreased from $2 \%$ to $0.4 \%$ that was equal to normal control group $(0.58 \%)$.
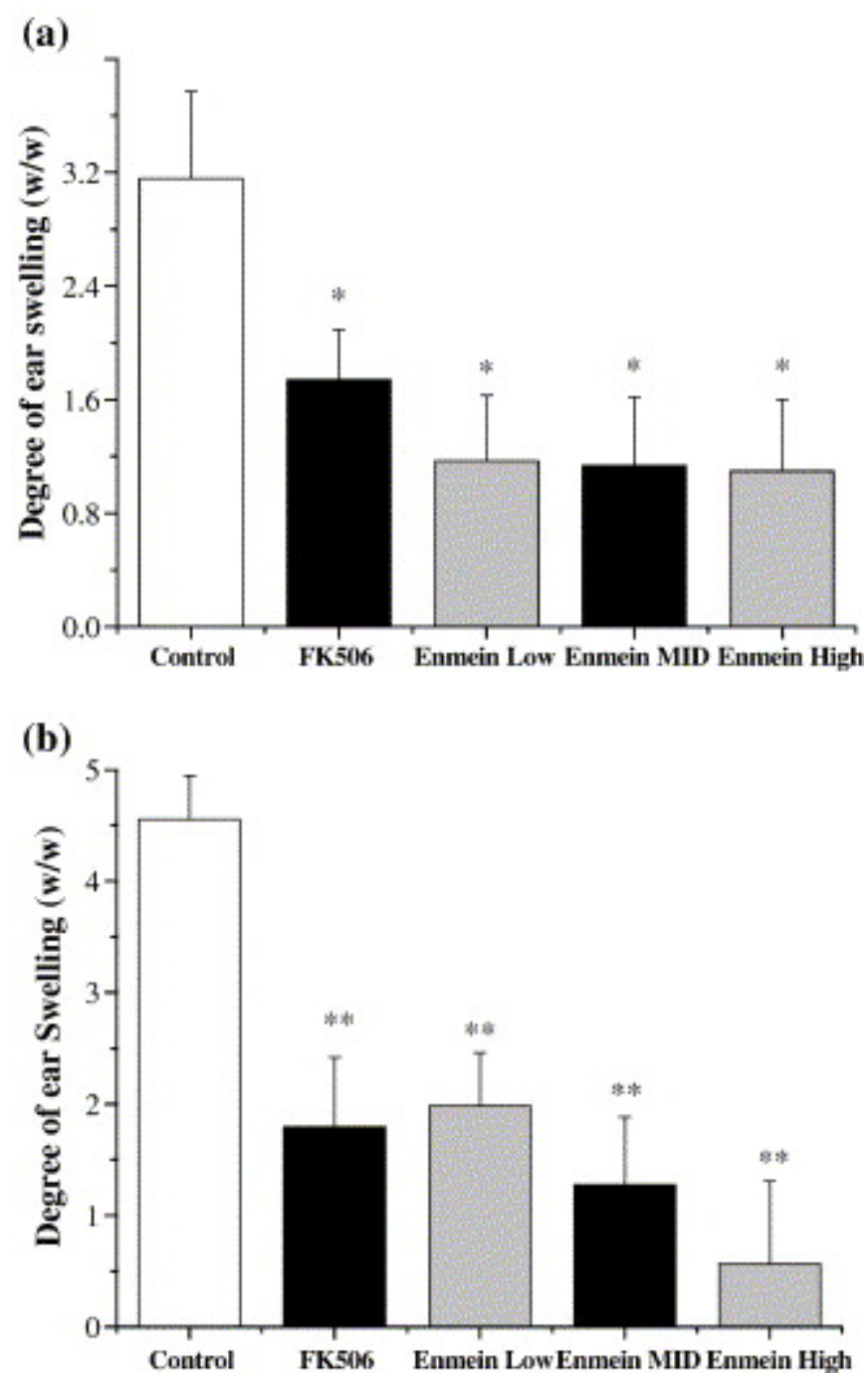

Figure 5: Effect of Enmein on mouse xylene-induced ear tumefaction. (a) It was observed that the xylene-induced ear swelling was significantly inhibited in female mice treated with FK506 $(1 \mathrm{mg} / \mathrm{kg})$ or Enmein separately at the dose of $6.52 \mathrm{mg} / \mathrm{kg}$ (LOW), $9.78 \mathrm{mg} / \mathrm{kg}$ (MID) and $13.04 \mathrm{mg} / \mathrm{kg}$ (HIGH) for 5 days. Inhibitive activity on ear swelling was noticed in Enmein groups that were more significant than FK506 group, compared with the control group. (b) It was observed that the xylene-induced ear swelling was significantly inhibited in male mice treated with FK506 or Enmein for 5 days. Compared with the control group, significantly inhibition on ear swelling was noticed in Enmein groups, in a dose-dependant manner. *Indicated significant difference between control and experimental, $p<0.05$; **indicated significant difference between control and experimental, $p<0.01$.

\section{Effect on cytokine production in vivo}

We directly tested Interleukin-2 levels in blood serum specimens from experimental animal model above through an ELISA procedure. In Fig. 6, the IL-2 concentration in high-dose-treated group $(13.04 \mathrm{mg} / \mathrm{kg})$ was lower than that in low-dose-treated group $(6.52 \mathrm{mg} / \mathrm{kg})$. Compared with control group, inhibition of enmein was significantly noticed in a dose-dependant manner. 
In a word, the increased level of inflammatory cytokine (Interleukin-2) was attenuated by the treatment of Enmein in vivo model.

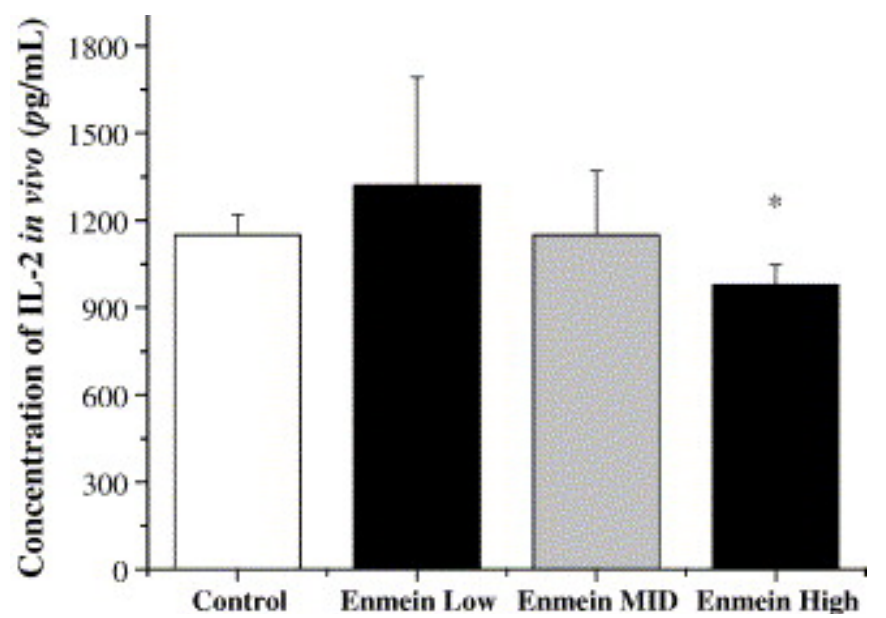

Figure 6: Effect of Enmein on cytokine production in vivo. We directly tested Interleukin-2 levels by an ELISA procedure. The concentration of IL-2 in low-dose-treated group $(6.52 \mathrm{mg} / \mathrm{kg})$ was higher than that in control group, while the concentration of IL-2 in high-dose-treated group $(13.04 \mathrm{mg} / \mathrm{kg})$ was much lower. Compared with control group, inhibition to Interleukin-2 was significantly noticed in a dose-dependant manner. *Indicated significant difference between control and experimental, $p<0.05$.

\section{DISCUSSION}

Cell proliferation was clearly accommodated within a chronic disease state such as rheumatoid arthritis (RA), where there was T cell infiltration of synovium and marked cytokine production [16], because stimulated T cells were able to support osteoclast formation [17]. According to the analysis of cell viability in vitro, I. serra extracts could significantly inhibit abnormally high splenic T lymphocyte (ConA-induced), while they had softer inhibitive activity on resting lymphocytes. We found that the efficiency of cellular immunosuppression was in the following order: Enmein > Nodosin > Epinodosin > Lasiodonin. Considering that the other extracts had lower therapeutic index $\left(\mathrm{IC}_{50} / \mathrm{EC}_{50}\right)$ than Enmein, we anticipated that the most appropriate drugs (Enmein), whose optimal concentration was higher than $\mathrm{EC}_{50}$ and lower than $\mathrm{IC}_{50}$ (11-17 $\mu \mathrm{g} / \mathrm{ml}$ ), would be beneficial. Some studies on Isodon genus extracts had discovered Enmein[5]. There was no information available for its immune activity, even though Enmein was the primary agent of $I$. serra. Among our extracts, it contained $3 \mathrm{mg}$ Enmein/100 g raw material but only $0.2 \mathrm{mg}$ Epinodosin/100 g raw material. In this paper, we discovered the immunosuppressive activity of Enmein as follows. Just as expected, an inhibitive activity was observed through the flow cytometry analysis assay. It showed that percentage of Enmein-treated cells (in 5.5-22 $\mu \mathrm{g} / \mathrm{ml}$ ranges) in the $\mathrm{S}$ and $\mathrm{G}_{2} / \mathrm{M}$ stage obviously decreased to the normal levels, while number of cells in the $G_{1}$ stage increased to the normal levels in a dose-dependant manner. This phenomenon implied that Enmein influenced cell cycle through inhibiting DNA replication. So it suggested that inhibitive activity of Enmein on T lymphocytes multiplication maybe related to interdiction of DNA replication in the $\mathrm{G}_{1}-\mathrm{S}$ stage and to regulation of cell mitosis cycle. Herein, we had primarily made the choice of the most appropriate compound (Enmein) from these entKaurene Diterpenoids. 
It was well known that rheumatoid arthritis was characterized by synoviocytes proliferation and articular cartilage tissue tumefaction. These pathological changes resulted from a profile of increased plasma levels of the proinflammatory cytokines Interleukin-2, which was thought to play an important role in the pathogenesis of RA [18]. Besides, T cells initiated the inflammatory cascade through secretion of IL-2 that was higher in the long-lasting RA [19]. Preliminary studies in our laboratory showed that $I$. serra extracts inhibited the proliferation of mitogenstimulated cultures of murine splenic T lymphocytes in vitro. Therefore, we tested production of IL-2 for the hypothesis that $I$. serra extracts altered T lymphocyte activation by modulating cytokines IL-2. In xylene-induced mouse ear swelling experiment, as we expected, Enmein (from $6.52 \mathrm{mg} / \mathrm{kg}$ to $13.04 \mathrm{mg} / \mathrm{kg}$ ) inhibited not only the mouse ear swelling extent, but also the production of IL-2 in murine blood serum dose-dependently. We supposed that it might be the important mechanism of immunosuppressive activity of Enmein. Such a unique modulation from Enmein might point to distinct signaling mechanisms for induction of IL-2.

In conclusion, from all the results we attained, it demonstrated that four ent-Kaurene Diterpenoids from I. serra had distinct immunosuppressive effect in vitro and in vivo. Enmein, which we selected for the most appropriate compound, was expected to be a promising drug for autoimmune disease such as rheumatoid arthritis. In order to promote research and development of $I$. serra extracts, more methods in pharmacological studies would be employed. For example, collagen-induced arthritis (CIA) and adjuvant induced Arthritis (AA) experimental animal models will be taken in our future investigation. As a promising immunosuppressive compound, more pharmacological activity and detailed mechanism of Enmein would be illuminated in future.

\section{ABBREVIATIONS \\ ConA Concanavalin A \\ IL-2 Interleukin-2 \\ RA rheumatoid arthritis \\ PBS phosphate buffered saline}

\section{ACKNOWLEDGEMENTS}

This work was supported by the Open Project Program of the State Key Laboratory of Bioreactor Engineering, East China University of Science and Technology. We were also grateful to our fellow workers: Xiaodong Zhang, Shuihua Zhang, Liyun Shen and Jihong Li, et al.

\section{REFERENCES}

[1] Luk JM, Chan JKY, Tam PKH. Differential suppression by Tripterygium wilfordii extracts (traditional Chinese medicine) in the allogeneic rat mixed lymphocyte reaction. Transplant Proc 2000;32:2055-7.

[2] Hayashi K, Hayashi T, Sun HD, Takeda Y. Contribution of a combination of ponicidin and acyclovir/ganciclovir to the antitumor efficacy of the herpes simplex virus thymidine kinase gene therapy system. Hum Gene Ther 2002;13:415-23.

[3] Hou AJ, Zhao QS, Li ML, Jiang B, Lin ZW, Sun HD, et al. Cytotoxic 7,20-epoxy entkauranoids from Isodon xerophilus. Phytochemistry 2001;58:179- 83. 
[4] Wu CY, Li HW. Flora Republican Popularis Sinicae, vol. 66. Beijing Science Press; 1997. p. 479.

[5] Li Baolin, Tian Xianhua. Enmein type diterpenoids from Isodon japonica. Phytochemistry 2001;58:543-6.

[6] Zhao Ai-Hua, Zhang Yan, Xu Zhao-hui, Liu Jianwen, Jia Wei. Immunosuppressive entkaurene diterpenoids from Isodon serra. Helv Chim Acta 2004;87:3160- 6.

[7] Pu LX, Zhang TM. Effects of triptolide on T lymphocyte function in mice. Acta Pharmacol Sin 1990;11:76-9.

[8] Zhang LX, Yu FK, Zheng QY, Fang Z, Pan DJ. Immunosuppressive and anti-inflammatory activities of tripterine. Pharm Sin 1990;25:573.

[9] Multi-glycoside of tripterygium wilfordii hook f. ameliorates proteinuria and acute mesangial injury induced by anti-thy1.1 monoclonal antibody. Nephron Exp Nephrol 2005;99:e121-9.

[10] Mouzaki A, Dai Y, Weil R, Rungger D. Cyclosporin A and FK506 prevent the derepression of the IL-2 gene in mitogeninduced primary T lymphocytes. Cytokine 1992;4:151- 60.

[11] Wermerskirchen Angela S, LaTocha Dorian H, Clarke Benjamin L. Adrenocortropid hormone controls concanavalin A activation of rat lymphocytes by modulating IL-2 production. Life Sci 2000;67:2117-87.

[12] Clarke BL, Gerhard BM, Blalock JE. Mitogen-stimulated lymphocytes release biologically active corticotrophin. Endocrinology 1993;132:983-8.

[13] Lee Dean A, Molinaro Giuseppe A. Activated peripheral T lymphocytes undergo apoptosis when cultured with monocytes activated by HLA class II ligation. Cell Immunol 2003;225:101- 12.

[14] Ao GZ, Zhang YH, Ji H, Deng G. Synthesis and anti-inflammatory activity of alphasubstituted p-(methanesulfonyl) phenylpropenamides. Yao Xue Xue Bao 2003;38:671- 6.

[15] Bhatia M. Tachykinins as therapeutic targets in inflammation. Current Medicinal Chemistry-Anti-Inflammatory and Anti-Allergy Agents, vol. 2. Bentham Science Publishers; 2003. p. 19- 27.

[16] Choy HS, Panayi GS. Cytokine pathways and inflammation in rheumatoid arthritis. Engl J Med 2001;344:907-16.

[17] Horwood Nicole J, Kartsogiannis Vicky, Quinn Julian MW, Romas Evangelos, Martin T. John, Gillespie Matthew T. Activated T lymphocytes support osteoclast formation in vitro. Biochem Biophys Res Commun 1999;265:144- 50.

[18] Cunnane G, Hummel KM, Youssef PP, Gay RE, Gay S, Bresnihan B. Synovial tissue protease gene expression and join erosions in early rheumatoid arthritis. Arthritis Rheum 2001;44:1744- 53.

[19] Mellish RW, O’Sullivan MM, Garrahan NJ, Compston JE. Ann Rheum Dis 1987;46:8306. 